\title{
NONLINEAR MODELLING AND VALIDATION OF AN AIRCRAFT GAS TURBINE ENGINE
}

\author{
Neophytos Chiras, Ceri Evans and David Rees \\ School of Electronics, University of Glamorgan, CF37 1DL Wales, UK \\ nchiras@glam.ac.uk
}

\begin{abstract}
In this paper the orthogonal estimation algorithm is used to estimate a NARMAX model for an aircraft gas turbine. The performance of the model is validated using a range of small and large signal tests and by examining the model's static and dynamic characteristics. The NARMAX representation allows the direct mapping to the frequency domain by computing the Higher Order Frequency Response Functions (HOFRF) thus allowing physical interpretation to be made. Copyright ${ }^{\circledR} 2001$ IFAC
\end{abstract}

Keywords: gas turbines, nonlinear models, modelling techniques, NARMAX models, frequency response.

\section{INTRODUCTION}

The modelling of gas turbine dynamics has been the subject of considerable study since the early days of jet propulsion. Gas turbines are now extensively used in aero, marine and industrial applications. With such widespread and increasing applications, the accurate modelling of such engines is an important issue.

Modelling of gas turbines is required both in the development and operational stages of an engine's life. Models can be used to predict the engine's performance, design and test the performance of the engine control systems and predict normal engine behaviour for on-line fault detection purposes. Models are usually validated against real data and in some cases a physical interpretation of the parameters can be made. This allows initial assumptions about the engine characteristics to be checked.

This paper deals with the nonlinear relationship between the fuel flow and shaft speed dynamics of a Rolls Royce Spey Mk202 aircraft gas turbine. Although no longer in service, the Spey possess the same characteristics, for control purposes as a modern engine such that of the EJ200 fitted to the Eurofighter (Dadd et al., 1996).

\section{THE GAS TURBINE}

A gas turbine is made up of three basic components: a compressor, a combustion chamber and a turbine. Air is drawn into the engine by the compressor, which compresses it and delivers it to the combustion chamber. Within the combustion chamber the air is mixed with fuel and the mixture is ignited, producing a rise in temperature and hence an expansion of gases. These gases are exhausted through the engine nozzle but first pass through the turbine, which is designed to extract sufficient energy from them to keep the compressor rotating.

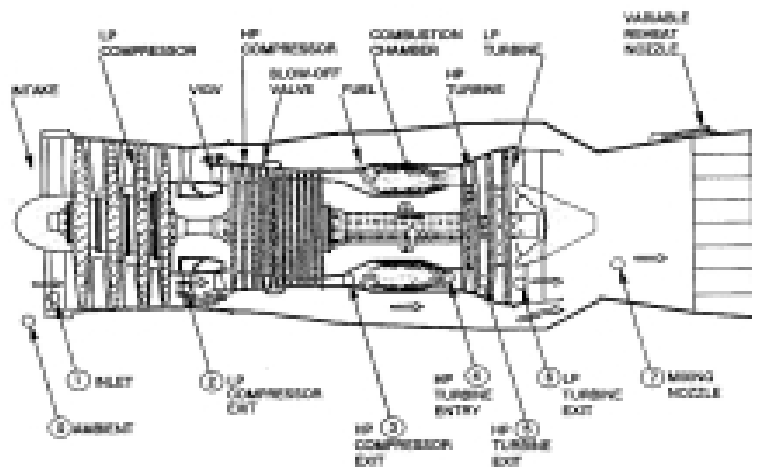

Fig. 1. Simplified schematic of a Rolls Royce Spey engine. 
A schematic diagram of the Rolls-Royce Spey engine tested in this work is shown in Fig. 1. It can be seen that both the compressor and the turbine are split into low pressure (LP) and high pressure (HP) stages. The HP turbine drives the HP compressor and the LP turbine drives the LP compressor. These are connected by concentric shafts, which rotate at different speeds, denoted $\mathrm{N}_{\mathrm{H}}$ and $\mathrm{N}_{\mathrm{L}}$. These shaft speeds are the primary outputs of a gas turbine, from which the internal engine pressures and thrust can be calculated.

Work conducted by Jackson (1988) for Rolls Royce plc, showed that the higher-order nonlinear thermodynamic models derived from the engine physics could be reduced to linear models of the same order as the number of engine shafts. The models were first linearised at a series of operating points, using small perturbations, and then a model reduction procedure was employed. Work done by Hill (1994) examined the application of time-domain methods to estimate discrete $z$-domain engine models. Even though linear discrete models with good input-output properties were estimated, problems with the application of time-domain techniques were reported (Hill, 1997).

More recently, teams at the universities of Glamorgan, Birmingham and Sheffield utilised the same data to investigate various identification techniques on a Rolls-Royce Spey engine. Data were gathered under sea-level static conditions at the Defence Evaluation \& Research Agency (DERA) at Pyestock. Multisine and inverse repeat maximum length binary sequences (IRMLBS) were used at amplitudes of up to $\pm 10 \%$ of the steady state fuel flow $\left(W_{f}\right)$. In addition higher-amplitude signals, such as triangular waves and three-level periodic signals with input amplitudes of up to $\pm 40 \% W_{f}$, were used. These caused the HP shaft speed to vary between $65 \%$ and $85 \%$ of its maximum value $\left(\% N_{H}\right)$.

Evans et al. $(1998,1999,2000)$ from the University of Glamorgan, used frequency techniques on the smallamplitude multisine and IRMLBS data to estimate linear models at different operating points. The errors due to noise and nonlinearities were assessed and found to be small for these small-signal models. The fact that the dc gains and the dynamics of these models change with operating point showed that the gas turbine is nonlinear, so the need is apparent for a more complete gas turbine description using nonlinear models.

In Evans et al. (2001) an extended least-squares algorithm with optimal smoothing was used by Norton, to identify time-varying transfer function models to represent large transient and nonequilibrium effects and provide a more detail insight into the slow thermal dynamics of the engine. In order to identify a model capable of representing the engine at all operating points, Rodriguez (1998a, 1998b) used a multiobjective genetic programming approach on the same data and allocated weights to various objectives, to assess their significance in the structure selection of Nonlinear AutoRegressive Moving Average with eXogenous inputs (NARMAX) models of the engine. A simple NARX model was identified which was able to represent both the small and large signal dynamics of the engine.

In this paper a NARMAX model for the engine is estimated using a forward-regression orthogonal estimation algorithm. The model is validated using a range of small and large signal tests and by comparing the model's static and dynamic behaviour with measured static data and estimated linear dynamics. The model is mapped to the frequency domain by computing the HOFRFs and the energy mechanisms revealed by the interaction of frequencies are examined.

\section{NARMAX MODELLING}

Leontaritis and Billings (1985) and Chen and Billings (1989), introduced the NARMAX approach as a means of describing the input-output relationship of a nonlinear system. The model represents the extension of the well-known ARMAX model to the nonlinear case, and is defined as

$$
\begin{aligned}
& y(k)=F\left(y(k-1), \ldots, y\left(k-n_{y}\right), u(k-1), \ldots,\right. \\
& \left.u\left(k-n_{u}\right), e(k-1), \ldots, e\left(k-n_{e}\right)\right)+e(k)
\end{aligned}
$$

where $F$ is a nonlinear function; $y(k), u(k)$ and $e(k)$ represent the output, input and noise signals respectively, and $n_{y}, n_{u}$, and $n_{e}$ are their associate maximum lags. The NARMAX representation constitutes a powerful tool for nonlinear modelling since it includes a family of other nonlinear representations such as block-structured models and Volterra series (Liu, 1988). In addition, the model is linear-in-the-parameters so that linear least-squares parameter estimation techniques can be easily applied. A well established procedure for structure selection of a NARMAX polynomial model is based on the error reduction ratio (ERR) defined in (Billings et al., 1989) as

$$
E R R_{i}=\frac{g_{i}^{2} \sum_{k=1}^{N} w_{i}^{2}(k)}{\sum_{k=1}^{N} y^{2}(k)}
$$

where $g_{i}$ are the coefficients and $w_{i}(k)$ are the terms of an auxiliary model constructed in such a way that the terms $w_{i}(k)$ are orthogonal over the data records. A forward-regression algorithm (Billings et al., 1989) is employed to select at each step the term with 
the highest ERR, in other words the term which contributes most to the reduction of the residual variance. The procedure is usually stopped using an information criterion such as the AIC, defined as

$$
A I C=N \log _{e}\left(\sigma_{\varepsilon}^{2}\left(\theta_{p}\right)\right)+k p
$$

where $\sigma_{\varepsilon}^{2}\left(\theta_{p}\right)$ is the variance of the residuals associated with a $p$-term model and $k$ is a penalising factor. This procedure was found to work very well when identifying polynomial NARMAX models and it does exclude the vast majority of insignificant candidate terms by working towards the reduction of the residual variance.

A concatenated data set of small signal tests is used for structure selection and parameter estimation. This is achieved by combining small-signal IRMLBS tests at different operating points to cover a range from $55 \%$ to $85 \% N_{H}$ as shown in Fig. 2. The forwardregression orthogonal estimation algorithm is applied to these data and the selected terms with their associated ERRs and the coefficients of the selected model are shown in Table 1.
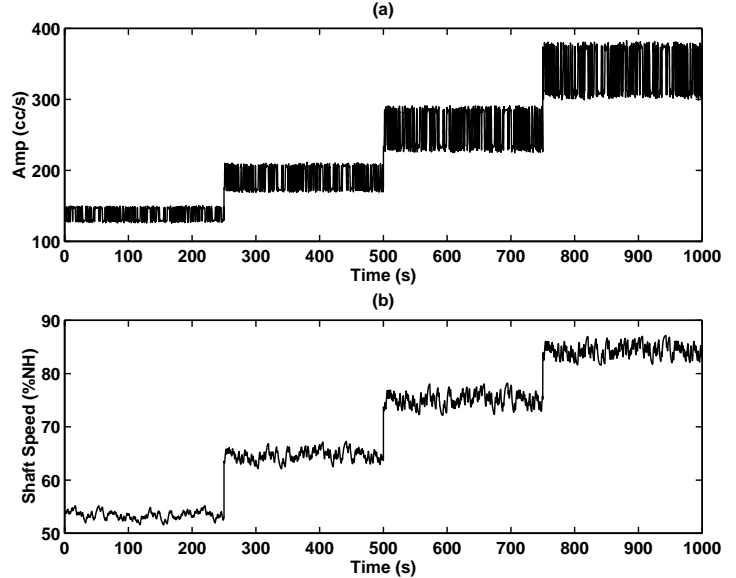

Fig. 2. Concatenated data set used for estimation (a) measured fuel flow (b) HP shaft response.

Table 1 Quadratic NARX Model 1.

\begin{tabular}{ccc}
\hline Model 1 terms & ERR $_{\mathrm{i}}$ & $\theta_{\mathrm{i}}$ \\
\hline$y(k-1)$ & $9.9 \mathrm{e}-1$ & 0.7453 \\
$u(k-1)$ & $1.9 \mathrm{e}-5$ & $4.083 \mathrm{e}-3$ \\
$y(k-2)$ & $1.3 \mathrm{e}-5$ & 0.2943 \\
$y(k-1) * y(k-2)$ & $3.2 \mathrm{e}-6$ & $-4.471 \mathrm{e}-4$ \\
constant & $8.8 \mathrm{e}-6$ & -1.1479 \\
\hline
\end{tabular}

The model quality can be assessed using higher order correlation functions (Billings and Voon, 1983), but no definite conclusion can be drawn unless crossvalidation is employed. Nonparametric validation is employed which consists of the simulation of the estimated model with different measured inputs and comparison of the results with the measured outputs.
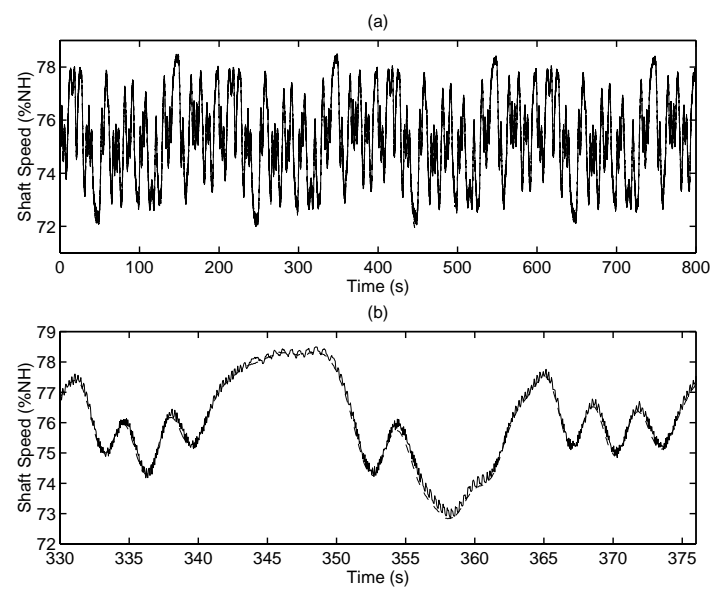

Fig. 3. Multisine HP shaft test. (a) complete signal (b) portion of the signal. Model 1 output (dashed) measured output (solid).

Fig. 3 shows a time-domain comparison between the model output and the measured gas turbine output for a small signal multisine test, where it can be seen that a very good match is achieved. Similar results are obtained when the nonlinear model is validated against small signal tests at different operating points, suggesting that Model 1 is suitable to be used in the place of the family of linear models previously estimated.

Model 1 is also validated against high-amplitude data. The next test, shown in Fig. 4, consists of a three-level sequence of period 100s with an input amplitude of $\pm 22 \% \quad W_{f}$. This test is the most demanding available engine test since it essentially consists of a series of positive and negative largeamplitude step responses. It can be again seen that the model shows a good response suggesting that the model is capable of representing the engine dynamics at high amplitudes.

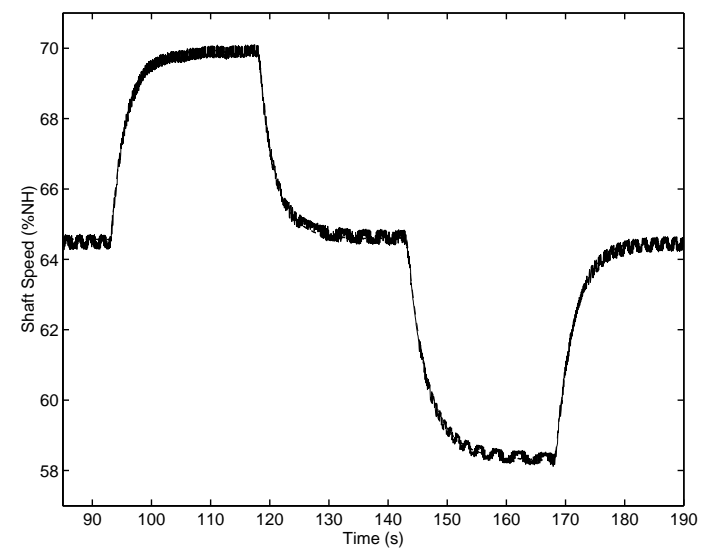

Fig. 4. Three-level HP shaft test. Model 1 output (dashed) measured output (solid).

An important test to assess the model performance is to examine its static behaviour at different operating points. The nature of the model did not allow the analytical derivation of the static gain in terms of the input (Aguirre et al., 1999), so the static behaviour of 
the model was derived through simulation and was compared with the static behaviour of the engine derived from measured data. It can be seen from Fig. 5 that the model is capable of modelling the static behaviour of the engine throughout the operating range.

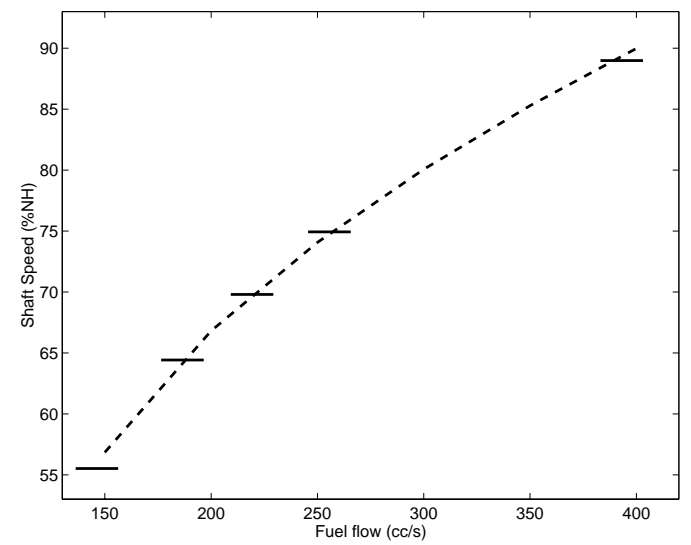

Fig. 5. Static relationship between shaft speed and fuel flow (solid bars) Model (dashed).

In order to study the dynamic behaviour of the model with operating point the model was simulated with multisine signals at the four operating points for which linear models were previously identified. Table 2 shows a comparison of the dominant modes identified from engine data using small signal tests and frequency domain techniques, and the dominant poles identified when simulation data was generated using the nonlinear model. It is again clear from the table that the local linearisation of the nonlinear model is very close to that of the engine at the different operating points.

Table 2. Linear models identified from engine data Vs local linear models obtained from Model 1.

\begin{tabular}{ccc}
\hline $\begin{array}{c}\text { Operating } \\
\text { point }\left(\% \mathrm{~N}_{\mathrm{H}}\right)\end{array}$ & $\begin{array}{c}\text { Estimated } \\
\text { models }\end{array}$ & $\begin{array}{c}\text { Locally linear } \\
\text { models }\end{array}$ \\
\hline 55 & -0.271 & -0.216 \\
65 & -0.394 & -0.373 \\
75 & -0.525 & -0.515 \\
85 & -0.800 & -0.651 \\
\hline
\end{tabular}

The structure selection procedure based on the ERR yielded a nonlinear model which is capable of modelling the fuel feed to shaft dynamics of the engine at both small and large amplitudes. During this work other different structures were also investigated which were found to have very good input-output properties. One of these models is shown in Table 3. This is a similar model as the one identified by Rodriguez (1998a) and by Chiras et al. (2000). The latter, used nonparametric analysis to limit the search space of models under consideration and then an exhaustive search to estimate the model shown in Table 3 . The performance of this model, referred to the remainder of this text as Model 2 was illustrated in Chiras et al. (2000) for both small and large amplitude inputs. It was shown that Model 2 is capable of representing the engine dynamics at small and large amplitudes.

Table 3 Quadratic NARX Model 2.

\begin{tabular}{cc}
\hline Model 2 terms & $\theta_{\mathrm{i}}$ \\
\hline constant & -1.1435 \\
$y(k-1)$ & 0.77135 \\
$y(k-2)$ & 0.26334 \\
$u(k-1)$ & $4.346 \mathrm{e}-3$ \\
$u(k-2)$ & $-2.746 \mathrm{e}-4$ \\
$y(k-1) * y(k-1)$ & $-4.455 \mathrm{e}-4$ \\
\hline
\end{tabular}

The performance of Model 2 is very similar to that of Model 1 estimated using the orthogonal estimation algorithm. This can be illustrated in Fig. 6 and Table 4 where a comparison of the static and dynamic behaviours of the two models is illustrated. From Fig. 1 it can be seen that the static behaviour of Model 1 is almost identical to that of Model 2. At the same time the dominant modes of the local linear models derived by the linearisation of the two models, are similar as shown in Table 4.

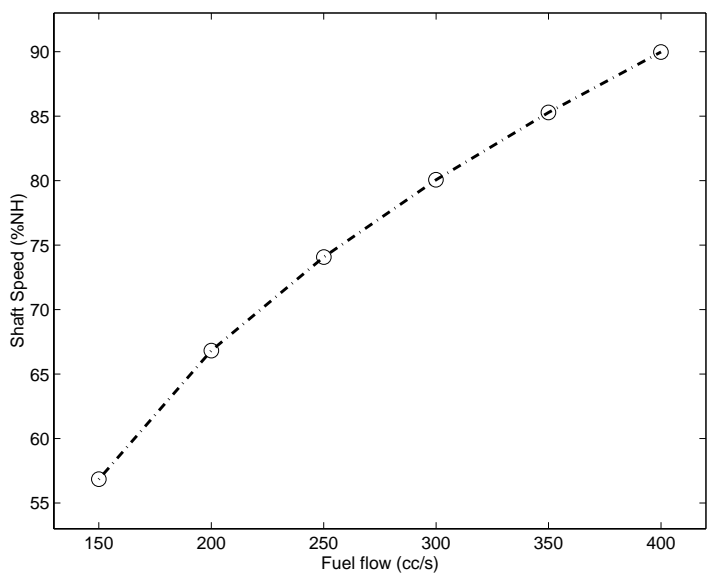

Fig. 6. Static relationship between shaft speed and fuel flow. Model 1 (circles) Model 2 (dashdot).

Table 4. Local linear models obtained from nonlinear Model 1 Vs local linear models obtained from nonlinear Model 2.

\begin{tabular}{ccc}
\hline $\begin{array}{c}\text { Operating } \\
\text { point }\left(\% \mathrm{~N}_{\mathrm{H}}\right)\end{array}$ & $\begin{array}{c}\text { Linear models } \\
\text { from Model 1 }\end{array}$ & $\begin{array}{c}\text { Linear models } \\
\text { from Model 2 }\end{array}$ \\
\hline 55 & -0.216 & -0.215 \\
65 & -0.373 & -0.369 \\
75 & -0.515 & -0.507 \\
85 & -0.651 & -0.637 \\
\hline
\end{tabular}

\section{FREQUENCY-DOMAIN ANALYSIS}

One way of analysing a discrete NARMAX model is by direct mapping to the frequency domain [Peyton Jones and Billings, 1989]. To this end, the probing method [Peyton Jones and Billings, 1989,1993] was 
used to derive the analytical expressions for the true first- and second-order generalised frequency response functions for both estimated models as shown in the Appendix. The amplitude of the true first-order (linear) FRF for Model 1 is shown in Fig. 7 where it can be seen that the engine is a low-pass system with a gain at zero frequency approximately equal to $-8 \mathrm{~dB}$. It should be noted here that provided the estimated model is the true model of the system the response shown in Fig. 7 is the true first-order frequency response function of the system, which in general is different from the biased estimate obtained from traditional spectral analysis.

The amplitude of the second-order HOFRF for Model 1 is shown in Fig. 8. Close inspection reveals the same type of low-pass characteristic as in the first-order case. The gain of the second-order HOFRF is found to be at least $70 \mathrm{~dB}$ down compared with the gain of the first-order FRF. This is of course significantly less that the first-order FRF hence the effect of the second-order HOFRF at the system output is not significant unless the input amplitude is large. It is clear from Fig. 8 that the effect of the second-order HOFRF is apparent at the peaks of the second-order HOFRF where the gain is at its maximum. This corresponds to the lines of frequency defined by $f_{1}=0, f_{2}=0$ and $f_{1}+f_{2}=0$ meaning that the quadratic nonlinearity in the system will only generate nonlinear contributions at the input frequencies and at dc.

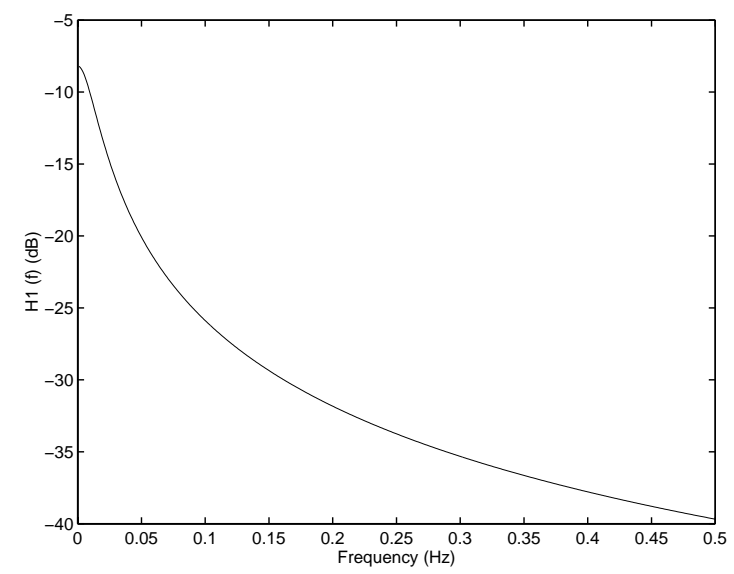

Fig. 7. First-order GFRF for Model 1.

The peaks on the second-order HOFRF at the lines of frequency defined by $f_{1}=0$ and $f_{2}=0$, indicate that there will be significant intermodulation effects on the output response if the signal contains a significant $\mathrm{dc}$ bias as in the engine case. This phenomenon is not limited to the dc case since these lines also represent other low frequency components, which will interact and create new harmonics at the output of the system. The peaks on the second-order HOFRF at the lines of frequency defined by $f_{1}+f_{2}=0$ indicate that there is a significant dc shift in the output response. This engine characteristic can be also observed from the time records in Fig. 2.
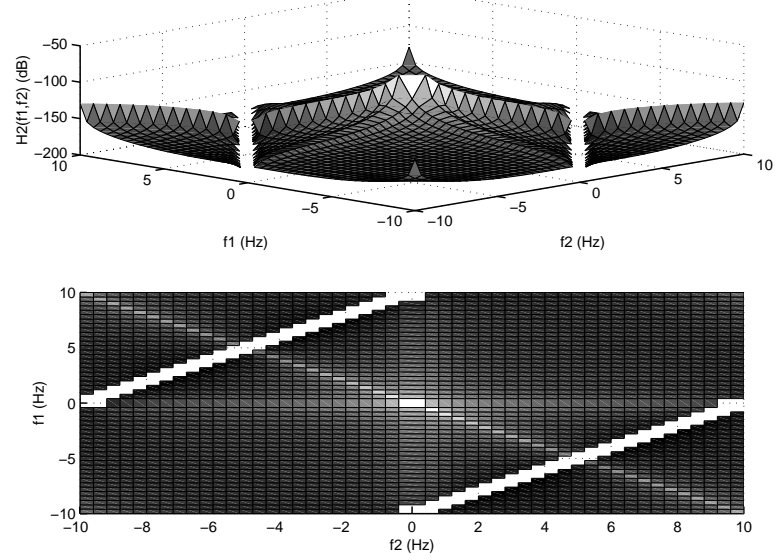

Fig. 8. Second-order HOFRF for Model 1.

An interesting feature, which is shown clearly in the plot, is the existence of two parallel deep gorges. This is because two exponential phasors cancel occasionally. In this case it can be easily seen from the numerator of $H_{2}\left(f_{1}, f_{2}\right)$ in the Appendix that when

$$
2 f_{1}+f_{2}=f_{1}+2 f_{2} \pm \frac{(2 m+1)}{2} \quad \mathrm{~m}=0,1,2, \ldots
$$

the numerator of $H_{2}\left(f_{1}, f_{2}\right)$ becomes zero. If the previous equation is rearranged to give

$$
\Delta f=f_{1}-f_{2}= \pm \frac{(2 m+1)}{2} \quad \mathrm{~m}=0,1,2, \ldots
$$

then the frequencies whose difference conforms with the above will cause a gorge. This gorge is a product of the $y(k-1) y(k-2)$ term in Model 1 since this term determines the numerator of $H_{2}\left(f_{1}, f_{2}\right)$.
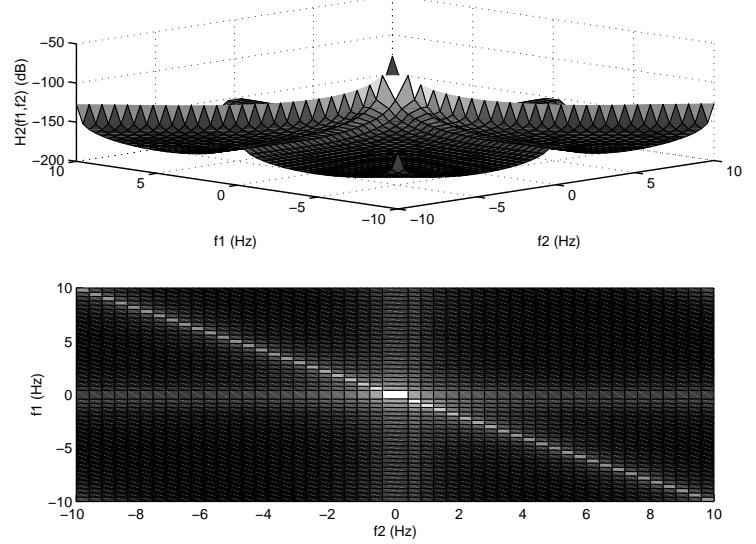

Fig. 9. Second-order HOFRF for Model 2.

Fig. 9 shows the amplitude of the second-order HOFRF of Model 2. It can be seen that the two models possess identical frequency characteristics except at the lines of frequency defined in equation (5). Since the models are almost equivalent in terms of their static and dynamic behaviour, and their HOFRFs are almost identical apart from the 
singularities in Model 1, then Model 2 is proposed as an appropriate model to approximate the nonlinear behaviour of the gas turbine.

\section{CONCLUSIONS}

In this paper a forward-regression orthogonal estimation algorithm was used to estimate a NARMAX model for an aircraft gas turbine. The performance of the model was validated using a range of small and large signal tests and by examining the model's static and dynamic behaviour. The estimated model was also compared with a previously estimated model. It was shown that the static and dynamic characteristics of the two models are almost identical.

The NARMAX representation allowed the direct mapping to the frequency domain by computing the HOFRFs. The analysis of these HOFRFs revealed the energy transfer mechanisms resulting from the nonlinearity, and facilitated the selection of Model 2 as the most appropriate nonlinear model for the gas turbine.

\section{ACKNOWLEDGMENTS}

This work was conducted on data gathered at the Defence Evaluation \& Research Agency at Pyestock with the support of Rolls Royce plc. The authors would like to thank all the staff involved.

\section{REFERENCES}

Aguirre, L.A., M.V. Correa, C.C.S. Cassini and G.G Rodriguez (1999). Static nonlinearities of polynomial and rational NARX models, Science and Engineering Journal, 8, 2, pp. 39-47.

Billings, S.A. and W.S.F. Voon (1983). Structure selection and model validity tests in the identification of nonlinear systems, IEE Proceedings, 130, 4, pp. 193199.

Billings, S.A., Chen, S. and M.J. Korenberg (1989). Identification of MIMO nonlinear systems using a forward-regression orthogonal estimator, Int. J. Control, 49, 6, pp. 2157-2189.

Chen, S. and S. A. Billings (1989). Representations of nonlinear systems: the NARMAX model, Int. J. of Control, 3, pp. 1013-1032.

Chiras, N., C. Evans, D. Rees (2000). Nonlinear Gas Turbine Computer modelling using NARMAX structure, Proceedings of the $17^{\text {th }}$ IEEE Instrumentation and Measurement Technology Conference, Baltimore, 3, pp 1278-1284.

Dadd, G.J., A. E. Sutton and A. W. M. Greig (1996). Multivariable control of military engines, AGARD Conference Proceedings No. 572 - Advanced AeroEngine Concepts and Controls, paper 28, pp. 1-12.

Hill, D.C. (1994). System identification of gas turbine engines, Ph.D. dissertation, University of Birmingham, School of Electronic and Electrical Engineering.

Hill, D.C. (1997). Identification of gas turbine dynamics: time-domain estimation problems, ASME Gas Turbine Conf., paper 97-GT-31, pp. 1-7.

Evans, C., D. Rees and D. Hill (1998). Frequency-domain identification of gas turbine dynamics, IEEE Transactions on Control Systems Technology, 6, 5, pp. 651-662.

Evans C., A. Borrell and D. Rees (1999). Testing and modelling gas turbines using multisine signals and frequency-domain techniques, ASME Journal of Engineering for Gas Turbine and Power, 121, pp. 451457.

Evans C., D. Rees and A. Borrell (2000). Identification of aircraft gas turbine dynamics using frequency-domain techniques, Control Engineering Practice, 8, 4, pp. 457-467.

Evans, C., P.J. Fleming, D.C. Hill, J.P. Norton, I. Pratt, D. Rees and K. Rodriguez-Vazquez (2001). Application of system identification techniques to aircraft gas turbine engines, Control Engineering Practice, 9, pp. 135-148.

Jackson, D. (1988). Investigation of state space architectures for engine models, Rolls Royce plc, Report TDR 9331.

Leontaritis, I. J. and S.A. Billings (1985). Input-output parametric models for nonlinear systems, Int. J. Control, 41, 2, pp. 311-341.

Liu, Y. P. (1988). Identification of Nonlinear Systems: The NARMAX Polynomial Model Approach", Ph.D. dissertation, University of Sheffield, Department of Automatic Control \& Systems Engineering, UK.

Peyton Jones, J.C. and S.A. Billings (1989). Recursive algorithm for computing the frequency response of a class of non-linear difference equation models, Int. $J$. Control, 50, 5, pp. 1925-1940.

Peyton Jones, J.C. and S.A. Billings (1993). Mean levels in nonlinear analysis and identification, Int. J. Control, 58, 5, pp. 1033-1052.

Rodriquez-Vazquez, K. and P.J. Fleming (1998a). Multiobjective genetic programming for gas turbine engine model identification, UKACC International Conference on Control, 2, 455, pp. 1385-1390.

Rodriquez-Vazquez, K. (1998b). Multiobjective evolutionary algorithms in non-linear system identification, Ph.D. Thesis, University of Sheffield, Department of Automatic Control \& Systems Engineering.

\section{APPENDIX}

First-and second-order transfer functions for Model 1.

$$
H_{1}(f)=\frac{4.08 \times 10^{-3} e^{-2 \pi j f}}{1-0.71 e^{-2 \pi j f}-0.27 e^{-2 \pi j 2 f}} \quad H_{2}\left(f_{1}, f_{2}\right)=\frac{-2.23 \times 10^{-4} H_{1}\left(f_{1}\right) H_{1}\left(f_{2}\right)\left(e^{-2 \pi j\left(f_{1}+2 f_{2}\right)}+e^{-2 \pi j\left(2 f_{1}+f_{2}\right)}\right)}{1-0.71 e^{-2 \pi j\left(f_{1}+f_{2}\right)}-0.27 e^{-2 \pi j\left(2 f_{1}+2 f_{2}\right)}}
$$

First-and second-order transfer functions for Model 2.

$$
H_{1}(f)=\frac{4.34 \times 10^{-3} e^{-2 \pi j f}-2.74 \times 10^{-4} e^{-2 \pi j 2 f}}{1-0.72 e^{-2 \pi j f}-0.26 e^{-2 \pi j 2 f}} \quad H_{2}\left(f_{1}, f_{2}\right)=\frac{-4.45 \times 10^{-4} H_{1}\left(f_{1}\right) H_{1}\left(f_{2}\right) e^{-2 \pi j\left(f_{1}+f_{2}\right)}}{1-0.72 e^{-2 \pi j\left(f_{1}+f_{2}\right)}-0.26 e^{-2 \pi j\left(2 f_{1}+2 f_{2}\right)}}
$$

